\title{
Noninvasive High-Frequency Shock Ventilation Based on Chest X-Ray Reconstruction Algorithm for Neonatal Respiratory Distress Syndrome
}

\author{
Xu Sang $\mathbb{D}^{\text {, }}$, Zhen Zhang $(\mathbb{D}$, Yumeng $W u(\mathbb{D}$, Wansheng Peng $\mathbb{i}$, and Xin Chen \\ Department of Pediatrics, The First Affiliated Hospital of Bengbu Medical College, Bengbu City 233004, Anhui Province, China \\ Correspondence should be addressed to Xin Chen; 0106046@yzpc.edu.cn
}

Received 14 May 2021; Accepted 7 July 2021; Published 19 July 2021

Academic Editor: Gustavo Ramirez

Copyright ( 2021 Xu Sang et al. This is an open access article distributed under the Creative Commons Attribution License, which permits unrestricted use, distribution, and reproduction in any medium, provided the original work is properly cited.

\begin{abstract}
Objective. To explore the use of the noninvasive high-frequency oscillatory ventilation and CPAP ventilation mode in the treatment of neonatal respiratory distress syndrome and to compare the treatment effect and the incidence of complications and whether it can reduce the time to go to the hospital and the number of hospital stays. Methods. Seventy-four children with RDS treated in hospital were selected and divided into the noninvasive high-frequency group (NHFV group, 36 children) and noninvasive positive pressure ventilation group (NCPAP group, 38 cases), and they were compared with the changes in arterial blood gas, the occurrence of complications, and the time on the machine before and after the operation on 12, 24, 48, and 72 hours. Results. In the NHFV group, $\mathrm{PO}_{2}, \mathrm{a} / \mathrm{APO}_{2}$, and $\mathrm{SaO}_{2}$ were higher than those in the NCPAP group at 12, 24, 48, and 72 $\mathrm{h}$ after the respiratory support was given, and the differences were statistically significant (all $P<0.05$ ). $\mathrm{PaCO}_{2}$ in the NHFV group was given respiratory support. After support, the results at 12, 24, 48, and $72 \mathrm{~h}$ were lower than those in the NCPAP group, and the difference was statistically significant (both $P<0.05$ ). The children in both groups were cured and discharged from the hospital, with air leakage, persistent pulmonary hypertension, and bronchopulmonary dysplasia; there were no statistically significant differences in the incidence of complications such as retinopathy, pulmonary hemorrhage, and intracranial hemorrhage $(P>0.05)$. The NHFV group had less tracheal intubation, operation time, and hospital stays than the NCPAP group. The differences were significant. Statistical significance was at $P<0.05$. Conclusion. Noninvasive high-frequency ventilation is effective in the treatment of RDS, and compared with the CPAP ventilation mode, it can reduce $\mathrm{CO}_{2}$ retention, increase the oxygenation index, and reduce time of operation and length of hospital stay in children with RDS. It is worthy of clinical promotion and application.
\end{abstract}

\section{Introduction}

Neonatal respiratory distress syndrome (NRDS) is a common severe lung disease in the neonatal period. It has the highest incidence and high mortality in preterm infants and requires respiratory support and intensive monitoring. For this reason, the key to solving this problem is to find a method that can improve clinical symptoms to effectively control the further deterioration of the disease [1]. In this study, we retrospectively analyzed children with neonatal respiratory distress syndrome who were hospitalized in hospital. At the same time, as symptomatic and supportive treatment, the addition of noninvasive high-frequency oscillatory ventilation (NHFOV) to assist breathing has achieved significant clinical results, and, compared with noninvasive positive pressure ventilation (NIPPV), it better assisted breathing, now reported as follows.

\section{Reconstruction Algorithm of Chest X-Ray}

The ordered subset maximum expectation method is an iterative image reconstruction algorithm developed based on the maximum likelihood expectation method (ML-EM). The traditional ML-EM is calculated as

$$
f^{(k)}(i, j)=\frac{f^{(k-1)}(i, j)}{\sum_{n, m} p(i, j, n, m)} \sum_{n, m} \frac{p(i, j, n, m) d(n, m)}{p\left(i^{\prime}, j^{\prime}, n^{\prime}, m^{\prime}\right) f^{(k-1)}\left(i^{\prime}, j^{\prime}\right)},
$$


where $(i, j)$ represents the pixel coordinates, $f(i, j)$ is the radioactive (strong) concentration represented by the ray, $(n, m)$ represents the spatial coordinates of the detector, and $d(n, m)$ is the number of photons detected by the $m$ pair detection unit when the angle is $\theta_{n}$ in the range of $0 \sim \pi$; $p(i, j, n, m)$ is the probability that the $(i, j)$ pixel falls into the $m$ pair detection unit when the angle is $\theta_{n}$. The ML-EM uses all the projection data to correct the value of each pixel of the reconstructed image during each iteration, and the reconstructed image is replaced only once. The OS-EM divides the projection data into $n$ subsets during each iteration. After each subset corrects the pixel values of the reconstructed image, the reconstructed image is updated once. All the subsets are calculated once, which is called an iteration process. In one iteration of the ML-EM, the reconstructed image is updated once, and in one iteration of the OS-EM, the reconstructed image is updated $n$ times, so the OS-EM has the effect of accelerating convergence. Usually, during each iteration of the traditional ML-EM or OS-EM algorithm, the following nonnegative conditions are automatically established:

$$
f^{(k)}(i, j) \geq 0
$$

The following constraint conditions are used to replace the nonnegative condition of (2):

$$
\begin{aligned}
& f^{(k)}(i, j) \geq f_{\text {low }}(i, j), \\
& f^{(k)}(i, j) \leq f_{\text {up }}(i, j) .
\end{aligned}
$$

In the above equation, $f_{\text {up }}(i, j)=$ const is the maximum possible value of the pixel, which is set according to prior knowledge, and $f_{\text {low }}(i, j)$ is calculated from the change in observation data.

In PET images, the so-called background refers to the distribution of radioactivity caused by body tissues such as muscle fat other than lesions and scattering. These distributions are generally spatially gradual. From a one-dimensional profile, this background is basically single; the distribution of the peak or bimodal structure can be well described by the second order (unimodal model) or third order (bimodal model). In addition, due to the different concentrations of radiopharmaceuticals involved in metabolism in different tissues of the human body, especially the lesions, they usually appear as isolated point sources relative to normal tissues. Therefore, it is assumed that the radioactive distribution of the fault to be reconstructed is composed of several isolated point sources and an overall background, and the background is slowly changing relative to the point source. In order to extract background information from the observed projection data, a nonlinear iterative fitting method is used, and the following low-order polynomials are selected:

$$
f_{\text {back }}(i, j)=\sum_{\alpha, \beta=0}^{\alpha+\beta \leq m_{r}} \alpha(\alpha, \beta) i^{\alpha} j^{\beta} .
$$

In the above formula, $\alpha$ is the fitting coefficient, $\alpha, \beta$ is a positive integer, and $\alpha+\beta$ is the maximum value. $m_{r}$ can be determined a priori based on the background knowledge of the reconstructed image. If $m_{r}=2$, the fitting background is a second-order polynomial. $m_{r}>3$ is generally not suitable, because, at this time, a part of highly metabolized (highly radioactive) tissue is also fitted. The theoretical projection data for this background is

$$
d_{\text {back }}(n, m)=\sum_{i, j} p(i, j, n, m) f_{\text {back }}(i, j) .
$$

Find the background count polynomial $f_{\text {back }}$ in the sense of least squares, and compare the calculated $d_{\text {back }}(m, n)$ with the actual measurement data $d(n, m)$, if it meets

$$
d(n, m)>d_{\text {back }}(n, m)+K \sqrt{d_{\text {back }}(n, m)} .
$$

Then, modify $d(n, m)$ to

$$
d(n, m)=d_{\text {back }}(n, m)+K \sqrt{d_{\text {back }}(n, m)},
$$

where $k \geq 0$ is the fitting parameter, and then a new $f_{\text {back }}$ is fitted from the new $d(n, m)$ and so on until $d(n, m)$ no longer changes. In essence, the process is to continuously subtract from the observation data components that deviate too much from the slowly changing low-order polynomials. These components mainly come from the point source of the mutation. The selection of $\alpha+\beta$ and $K$ is related to the prior knowledge. The larger the value of $K$, the greater the impact of the background on the reconstructed image. On the contrary, the smaller the value of $K$, the more the impact of the background on the reconstructed image. It can be selected based on the prior knowledge of the tomographic image. If it is known that there may be obvious lesions, the value of $K$ can be selected to be smaller. In order to achieve higher spatial resolution, the selection of more model parameters based on prior knowledge is inevitable. Reasonably select final $f_{\text {back }}$ as the lower limit $f_{\text {low }}$.

\section{Materials and Methods}

3.1. General Information. A retrospective analysis was made on the NRDs neonates admitted to our hospital during the period from January 2018 to December 2019. The diagnostic criteria for NRDs are the 4th edition of "Practical Neonatal" edited by Shao Xiaomei and others. Diagnostic criteria included the following: (1) symptoms of dyspnea, including shortness of breath, number of breaths $>60$ breaths $/ \mathrm{min}$, accompanied by groaning and inspiratory triconcavity signs, and dyspnea presenting progressively; and (2) chest X-rays suggesting changes in RDS. Children with congenital heart disease, respiratory malformations, gastrointestinal malformations, and infectious diseases were excluded [2].

This study was approved by the hospital ethics committee and the family's written informed consent. A total of 74 children with NRDs who met the inclusion criteria were divided into noninvasive high-frequency group (NHFV group, 36 children) and noninvasive positive pressure ventilation group (NCPAP group, 38 children), of which there were 42 males and 32 females, with a ratio of 1.3 to 1.0. There were 25 children with natural childbirth and 49 
children with cesarean section. The Apgar Neonatal score ranged from 1 to 3 and 12 to 4 from 1 to 7 after birth. It was divided into 34 cases, 8 to 10 points, 28 cases; 8 full-term infants, 66 preterm infants (gestational age $<35$ weeks and 57 cases), of which birth weight was $<1,000 \mathrm{~g}, 8$ cases, 1 to $1,000 \mathrm{~g}$, 1 to $500 \mathrm{~g}, 19$ cases, 1 ; There were 32 cases from $500 \mathrm{~g}$ to 2 to $500 \mathrm{~g}$ and 15 cases from 2 to $500 \mathrm{~g}$. Congenital malformation, severe heart, liver and kidney dysfunction, immune dysfunction, and genetic metabolic diseases were all excluded. The two groups of children were compared in terms of gender, gestational age, body weight, blood gas analysis, and severity of the disease. There was no statistical difference. Significance of learning was at $P>0.05$. The information is comparable.

\subsection{Method}

3.2.1. Intervention Countermeasures. All children with RDS were instilled with pig lung phospholipid injection (Gursu, Casey Pharmaceutical Co., Ltd.) at $200 \mathrm{mg} / \mathrm{kg}$ trachea under tracheal intubation. After tracheal intubation, noninvasive high-frequency oscillating ventilation or noninvasive positive pressure ventilation was given, and the ventilator parameters were adjusted according to blood gas analysis results and clinical manifestations.

3.2.2. Ventilation Mode. (1) For the NHFOV mode ventilator (Germany, model: 3090medin-CNO), the NHFOV initial adjustment parameters were as follows: $\mathrm{FiO}_{2}$ (oxygen concentration): $30 \%$ to $40 \%$, MAP (average airway pressure): 8 to $12 \mathrm{~cm} \mathrm{H}_{2} \mathrm{O}\left(1 \mathrm{cmH}_{2} \mathrm{O}=0\right.$. $(98 \mathrm{kPa})$, with a frequency of 7 to $12 \mathrm{~Hz}$, and an amplitude of 2 to 3 times that of MAP. Specifically, it is based on seeing a good thoracic oscillation. The chest radiograph shows lung capacity in the 8th to 9th intercostal space. (2) For the NCPAP mode ventilator, the NCPAP initial adjustment parameters were as follows: $\mathrm{FiO}_{2}$ : 0.30 to 0.40 , PEEP: 1 to $2 \mathrm{~cm} \mathrm{H}_{2} \mathrm{O}$, and flow rate: 8 to $10 \mathrm{~L} / \mathrm{min}$. The parameters adjustment was as follows: PEEP: $1 \sim 2 \mathrm{~cm} \mathrm{H}_{2} \mathrm{O}$, $\mathrm{FiO}_{2}$ : $50 \%$, evacuation NCPAP indication: PEEP: $2 \mathrm{~cm} \mathrm{H}_{2} \mathrm{O}$, $\mathrm{FiO}_{2}<30 \%$, no shortness of breath, $\mathrm{SO}_{2}>90 \%$ [3].

3.2.3. Chest X-Ray Diagnosis. All newborns underwent lowdose spiral chest X-ray for diagnosis. Eight-row spiral chest $\mathrm{X}$-ray with GE LightSpeed was selected for multichannel data acquisition. When scanning the newborn, the parameters are set to $80 \mathrm{kV}$ and $40 \mathrm{~mA}$, and the pitch is 3.0 to reduce the amount of radiation to the newborn. Low-dose spiral chest X-ray was used to scan the newborn's chest quickly, and the image was thinned and processed.

\subsubsection{Criteria for Judging Effective Treatment Criteria.} The effective treatment criteria are as follows: (1) clinical manifestations without shortness of breath; (2) percutaneous oxygen saturation maintained between $90 \%$ and $95 \%$; (3) arterial blood gas: $\mathrm{PaO}_{2}$ maintained at 60 to $80 \mathrm{mmHg}$ and $\mathrm{PaCO}_{2}$ maintained at 40 to $60 \mathrm{mmHg}$; (4) IOI $>300$. The ineffective treatment criteria are as follows: (1) MAP (mean airway pressure) $>14 \mathrm{~cm} \quad \mathrm{H}_{2} \mathrm{O}$ or $\mathrm{FIO}_{2}$ (oxygen concentration) $>50 \%$ able to maintain normal blood oxygen saturation; (2) $\mathrm{PaCO}_{2}$ in arterial blood gas $>70 \mathrm{mmHg}$; (3) frequent apnea: at least 6 episodes in $24 \mathrm{~h}$ or need $>2$ resuscitation airbag positive pressure ventilation to maintain normal oxygen saturation. Evacuation criteria are as follows: ventilator parameters reduced to a minimum, no signs of aspiration, and percutaneous oxygen saturation $>90 \%$.

3.2.5. Observation Indicators. Observation indicators are as follows: arterial blood gas changes, complications, duration of hospitalization with HFOV or CPAP, and length of hospital stay before and after treatment at $1 \mathrm{~h}, 12 \mathrm{~h}, 24 \mathrm{~h}$, $48 \mathrm{~h}$, and $72 \mathrm{~h}$. The neonatal lung field transparency, diaphragm, heart shadow, granular shadow, bronchial inflation signs, and other combined signs were observed.

3.3. Statistical Methods. The SPSS Q17.0 statistical software was used. The measurement data was expressed as $\chi \pm s$. The multiple tests were compared using the $F$ test, the two tests were compared using the $t$-test, and the count data were tested using the $\chi^{2}$ test. $P<0.05$ was considered statistically significant.

\section{Results}

4.1. Comparison of $\mathrm{PO}_{2}, \mathrm{PaCO}_{2}, a / A P O_{2}$, and $\mathrm{SaO}_{2}$ in Two Groups of Children. $\mathrm{PO}_{2}, \mathrm{a} / \mathrm{APO}_{2}$, and $\mathrm{SaO}_{2}$ in the NHFV group were significantly higher than those in the NCPAP group after $12,24,48$, and $72 \mathrm{~h}(P<0.05)$. At $12 \mathrm{~h}, 24 \mathrm{~h}, 48 \mathrm{~h}$, and $72 \mathrm{~h}, \mathrm{PaCO} 2$ levels in the NHFV group after receiving respiratory support were lower than those in the NCPAP group. All were lower than the NCPAP group (all $P<0.05$ ), and the differences were statistically significant; see Tables 1 and 2 .

4.2. Comparison of the Incidence of Complications between Two Groups of Children. Both groups of patients reached the recovery standard when discharged from hospital. In terms of the incidence of complications, such as air leakage, persistent pulmonary hypertension, bronchopulmonary dysplasia, retinopathy, pulmonary hemorrhage, and intracranial hemorrhage, the differences were not statistically significant $(P>0.05)[4]$, as shown in Table 3.

4.3. Comparison of Tracheal Intubation, Operation Time, and Length of Hospital Stay between Two Groups of Children. The tracheal intubation, the time of operation, and the length of hospital stay were lower in the NHFV group than in the NCPAP group, and the differences were statistically significant $(P<0.05)$. See Table 4.

\subsection{Chest X-Ray Diagnosis Comparison}

4.4.1. Lung Field Transparency. Six neonates were grade I lung field, and the change in transmittance was not prominent; 9 cases of neonates were grade II lung field, and the transparency was partially reduced; 11 cases were grade 
TABle 1: Comparison of $\mathrm{PO}_{2}$ and $\mathrm{PaCO}_{2}$ in two groups of children.

\begin{tabular}{ccccccc}
\hline & Time (h) & & 0 & 12 & 24 & 48 \\
\hline & NHFV group & $44.6 \pm 3.7$ & $55.6 \pm 3.9$ & $62.4 \pm 4.5$ & $70.5 \pm 6.6$ & $85.6 \pm 7.2$ \\
$\mathrm{PO}_{2}(\mathrm{mmHg})$ & NCPAP group & $44.0 \pm 3.6$ & $51.5 \pm 3.5$ & $58.1 \pm 4.0$ & $63.4 \pm 5.8$ & $76.6 \pm 6.5$ \\
& & 0.86 & 4.39 & 5.52 & 8.35 & 9.56 \\
& $P$ & $>0.05$ & $<0.05$ & $<0.05$ & $<0.05$ & $<0.05$ \\
\hline & NHFV group & $56.68 \pm 5.9$ & $48.3 \pm 4.6$ & $44.3 \pm 4.3$ & $40.7 \pm 3.9$ & $35.7 \pm 3.5$ \\
$\mathrm{PaCO}_{2}(\mathrm{mmHg})$ & NCPAP group & $57.0 \pm 5.9$ & $52.0 \pm 4.9$ & $48.8 \pm 4.5$ & $44.5 \pm 4.0$ & $29.5 \pm 3.9$ \\
& $\mathrm{t}$ & 0.97 & 4.25 & 4.32 & 5.92 & $<.1$ \\
& $P$ & $>0.05$ & $<0.05$ & $<0.05$ & $<0.05$ & $<0.05$ \\
\hline
\end{tabular}

TABle 2: Comparison of $\mathrm{a} / \mathrm{APO}_{2}$ and $\mathrm{SaO}_{2}$ in two groups of children.

\begin{tabular}{ccccccc}
\hline & Time $(\mathrm{h})$ & 0 & 12 & 24 & 48 & 72 \\
\hline & NHFV group & $0.21 \pm 0.09$ & $0.38 \pm 0.12$ & $0.48 \pm 0.17$ & $0.61 \pm 0.24$ & $0.76 \pm 0.38$ \\
$\mathrm{a} / \mathrm{APO}_{2}$ & NCPAP group & $0.22 \pm 0.09$ & $0.32 \pm 0.09$ & $0.42 \pm 0.11$ & $0.52 \pm 0.18$ & $0.70 \pm 0.31$ \\
& $t$ & 0.63 & 5.54 & 5.72 & 7.29 & $<.05$ \\
& $P$ & $>0.05$ & $<0.05$ & $<0.05$ & $<0.05$ \\
\hline & NHFV group & $48.4 \pm 5.7$ & $60.9 \pm 6.5$ & $75.5 \pm 8.0$ & $86.8 \pm 9.6$ & $92.6 \pm 9.9$ \\
$\mathrm{SaO}_{2}(\%)$ & NCPAP group & $48.6 \pm 5.6$ & $55.8 \pm 6.0$ & $65.9 \pm 7.3$ & $72.5 \pm 7.8$ & $85.1 \pm 5.7$ \\
& $t$ & 1.05 & 5.48 & 6.28 & 9.45 & $<.62$ \\
& $P$ & $>0.05$ & $<0.05$ & $<0.05$ & $<0.05$ & $<0.05$ \\
\hline
\end{tabular}

TABLE 3: Comparison of the incidence of complications between the two groups of children.

\begin{tabular}{lccr}
\hline Group & NHFV group & NCPAP group & $\chi_{2}$ \\
\hline Number of cases & 36 & 38 & $P$ \\
Air leak & 1 & 0 & 1.07 \\
Persistent pulmonary hypertension & 4 & 5 & 0.07 \\
Bronchopulmonary dysplasia & 1 & 2 & 0.05 \\
Retinopathy & 2 & 3 & $>0.05$ \\
Pulmonary hemorrhage & 0 & 1 & $>0.05$ \\
Intracranial hemorrhage & 2 & 1 & $>0.05$ \\
\hline
\end{tabular}

TABle 4: Comparison of tracheal intubation, operation time, and length of hospital stay in two groups of children.

\begin{tabular}{|c|c|c|c|c|}
\hline Group & NHFV group & NCPAP group & $t / \chi^{2}$ & $P$ \\
\hline Number of cases & 36 & 38 & & \\
\hline Tracheal intubation & 5 & 14 & 5.1 & $<0.05$ \\
\hline On time & $3.8 \pm 1.8$ & $5.2 \pm 2.5$ & 7.85 & $<0.05$ \\
\hline The number of days in hospital & $11.3 \pm 3.6$ & $15.6 \pm 5.2$ & 9.83 & $<0.05$ \\
\hline
\end{tabular}

III lung fields, and the transmittance was significantly reduced; 4 cases were grade IV lung field, showing uniform dense shadow, called white lung, as shown in Figure 1 [5].

4.4.2. Horizontal and Heart Shadow Conditions. 7 neonates were grade I with clear diaphragm and heart shadow outline; 9 cases of neonates were grade II with diaphragm and heart shadow outline clear; 11 cases were grade III with diaphragm and heart shadow outline unclear; three neonates were grade IV, and the heart contour of diaphragm was not seen, as shown in Figure 2.

4.4.3. Granular Shadow. 8 cases were grade I, and the lesions were localized in the lower field of both lungs with clear edges; 9 cases were grade II, and some granular shadows were fused into a slice; 13 cases were grade III; most of the granular shadows were fused, and the border was relatively clear, as shown in Figure 3.

4.4.4. Bronchial Inflation Signs. Six neonates were grade I, and no obvious bronchial inflation signs appeared; 9 cases of neonates were grade II, and some bronchial inflation signs were found; 15 cases of neonates were grade III, and there were extensive bronchial inflation signs, manifested as dry branches, beyond the silhouette of the heart.

4.4.5. Other Signs of Complications. 3 neonates had gas accumulation in the lower esophagus, 3 neonates had pneumothorax, 7 neonates had intracranial hemorrhage, 7 

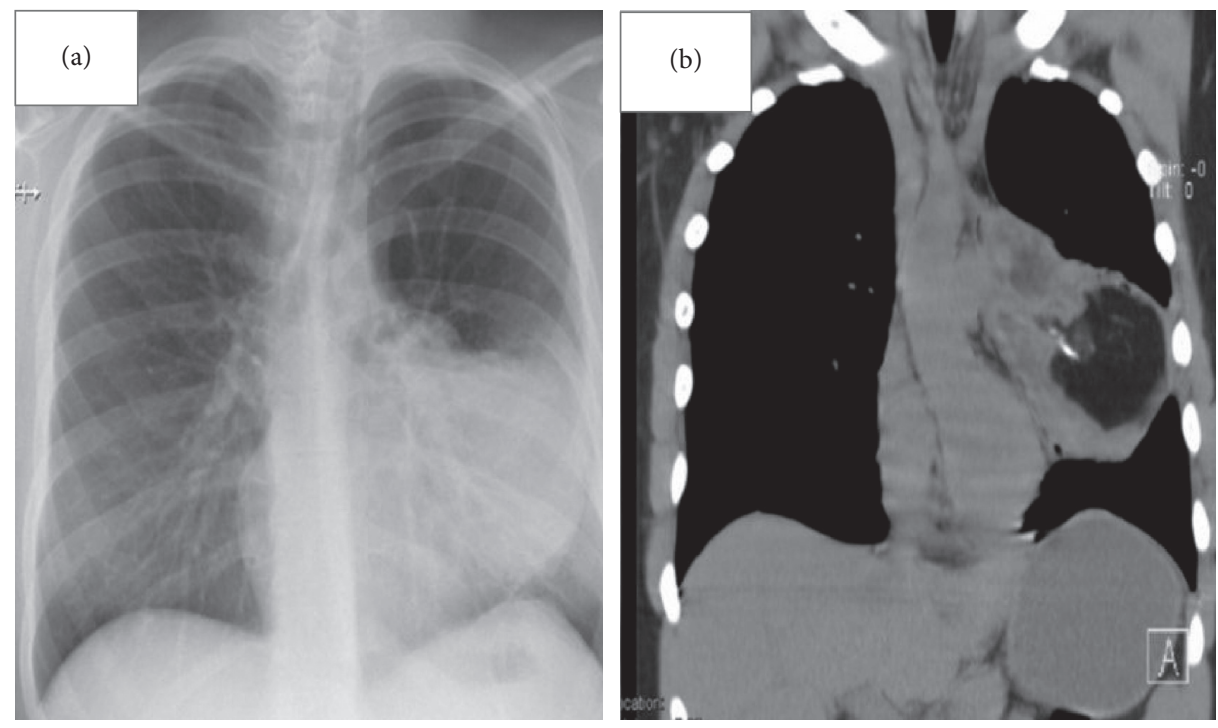

Figure 1: Lung field brightness.

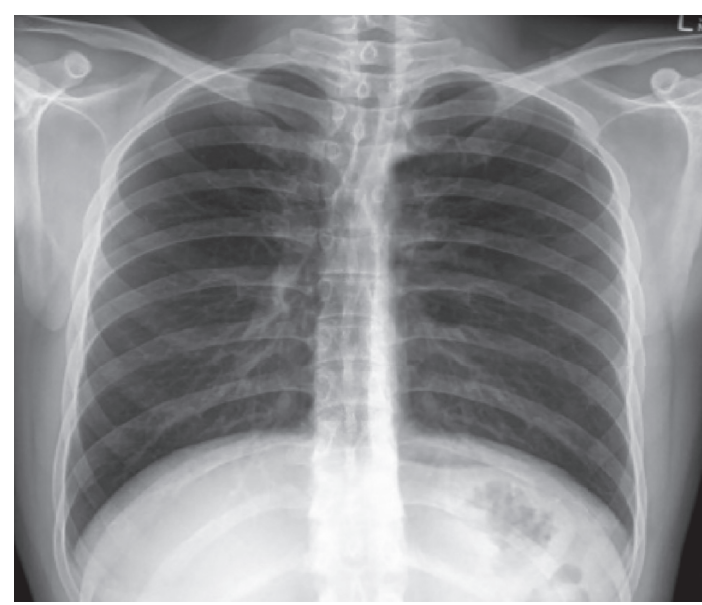

Figure 2: Chest X-ray of the heart and heart shadow.

neonates had intestinal gas accumulation, and 3 neonates had heart shadow increases [6].

\section{Discussion}

5.1. Effect of High-Frequency Oscillatory Ventilation on Neonatal Respiratory Distress. Neonatal respiratory distress syndrome is caused by pulmonary alveolar collapse due to lack of pulmonary surfactant (PS). It is more common in term infants than in preterm infants, selective cesarean section, and diabetic mothers. Most of them have progressive dyspnea, moaning, aspiration signs, bruising, and respiratory failure within a few hours after birth. It is a serious lung disease during the neonatal period and is one of the main causes of neonatal death. There are study reports that show that the mortality rate of neonatal respiratory distress syndrome in China is as high as $40 \%$ to $60 \%$, with poor prognosis and high incidence of sequelae. It has attracted much attention from neonatal physicians and families of children. Therefore, in addition to timely application of PS, seeking a timely correct way of giving life support is very important to save the lives of children and improve the prognosis. Invasive mechanical ventilation has been widely used in the treatment of neonatal respiratory distress syndrome. Although the ventilation effect is good, the technical requirements are high, invasive operation of tracheal intubation is required, the physical damage to the child is large, and the respiratory tract management is difficult, which has increased nursing workload; prolonged invasive ventilation is prone to a variety of serious complications such as invasive ventilation-related lung injury, ventilator-associated pneumonia, and chronic lung disease.

The working principle of CPAP is to provide a certain positive airway pressure in both inspiratory and expiratory phases in children with spontaneous breathing ability, thereby preventing airway collapse, increasing transpulmonary pressure and functional residual volume, and making airway diameter increase and improving lung compliance and ventilation/blood flow ratio, reduce the consumption of PS, and cause thorax vibration, and, in order to increase the safety of clinical applications, the CPAP is equipped with an air-oxygen mixed humidification device, flow monitoring, pressure monitoring, and alarm system, but there are certain adverse reactions to CPAP, such as carbon dioxide retention, hypoxemia, and the impact on the gastrointestinal tract [7].

In recent years, the reason why noninvasive high-frequency oscillating ventilation has been more and more popular among neonatal physicians is that it is a brand-new noninvasive ventilation mode. NHFOV has CPAP (noninvasive connection, increasing functional residual gas volume, allowing improved oxygenation). In addition to the characteristics, it also has the advantages of no breathing synchronization, good carbon dioxide scavenging ability, small volume injuries, and barotrauma. At present, there have been reports in the foreign literature that NHFOV may be used to avoid mechanical ventilation and its complications and as a respiratory support after weaning. The 


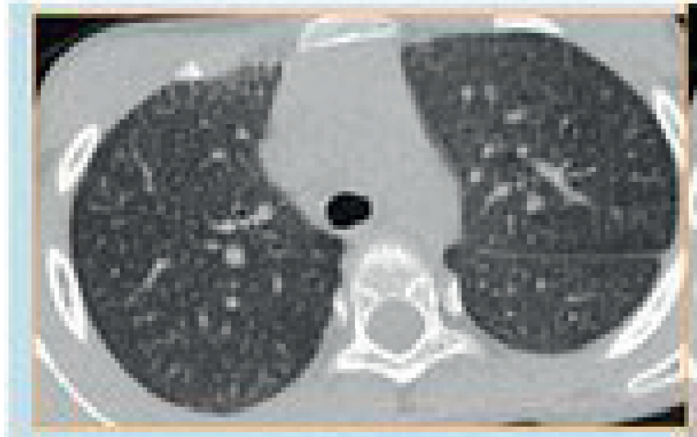

(a)

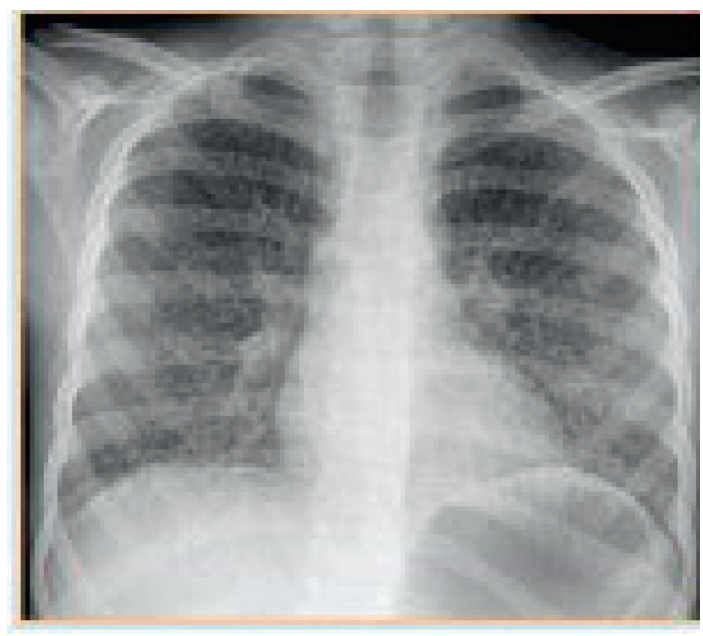

(c)

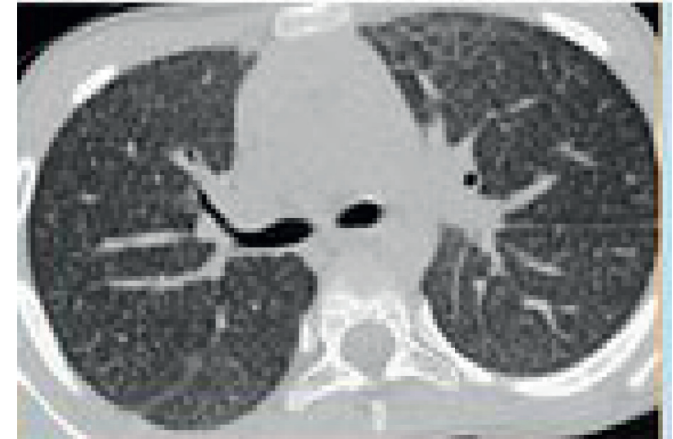

(b)

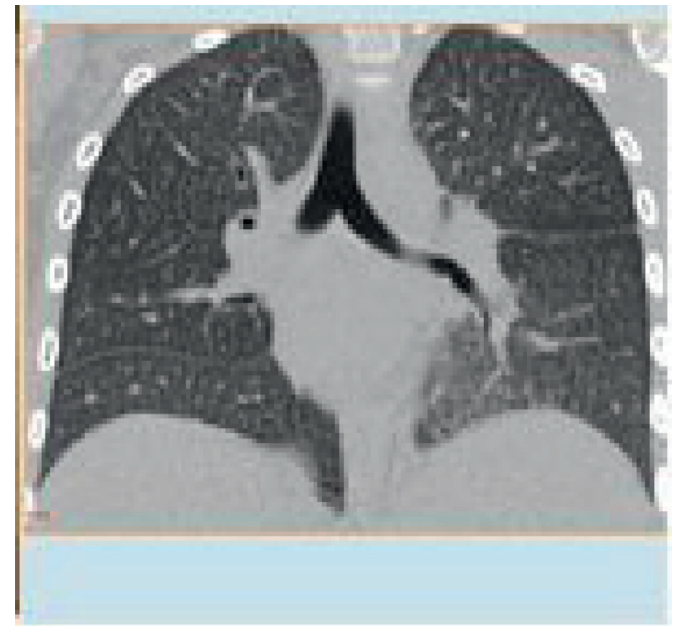

(d)

FIgURE 3: Granular shadows.

transition is effective, and the domestic research literature in this area is still scarce. The results of this study showed that $\mathrm{PO}_{2}, \mathrm{a} / \mathrm{APO}_{2}$, and $\mathrm{SaO}_{2}$ in the NHFV group were higher than those in the NCPAP group after $12,24,48$, and $72 \mathrm{~h}$ after receiving respiratory support. The difference between the NHFV group and the NCPAP group is statistically significant, indicating that noninvasive high-frequency oscillatory ventilation's assisted breathing is better than CPAP's assisted breathing in preventing $\mathrm{CO}_{2}$ retention and has higher oxygenation performance, which is consistent with foreign research reports. Its working principle may be the following: high frequency oscillation ventilation prevents alveolar collapse through high breathing frequency and small tidal volume, so that the expanded alveoli have enough gas to exchange and reduce or avoid tissue damage to children's lungs caused by high pressure and high ventilation. After the two groups of children met the clinical rehabilitation standards and were discharged, the incidences of complications were air leakage, persistent pulmonary hypertension, bronchopulmonary dysplasia, retinopathy, pulmonary hemorrhage, intracranial hemorrhage, etc. There was no significant difference in the incidence of complications between the two groups. The significance indicates that both noninvasive high-frequency oscillating ventilation and CPAP are safer ventilation modes, but it is not ruled out that the small sample size is related to further research.
However, the NHFV group was less than the CPAP group in tracheal intubation, operation time, and length of hospital stay, and the differences were statistically significant $(P<0.05)$. Therefore, noninvasive high-frequency oscillatory ventilation is more advantageous in treating RDS. However, whether it can be used as a remedy after failure of continuous noninvasive positive pressure ventilation mode remains to be further studied [8].

5.2. Chest X-Ray Diagnosis of Neonatal Respiratory Distress. At present, there are relatively few reports on the use of chest low-dose scanning technology in pediatric examinations. Chest X-ray examinations can cause radiation damage to the subject's body. Compared to adults, children have higher sensitivity to radiation damage. Studies show that the difference between children and adults is 2 to 3 times, and the sensitivity to the risk of cancer is 10 times higher in children than in adults $[3,4]$. Therefore, when chest X-ray examinations are used in pediatric examinations, it is necessary to not only obtain satisfactory image quality but also minimize radiation. Some studies have suggested that the exposure of $35 \mathrm{~mA}$ for pediatric chest X-ray examination is more appropriate. Currently, due to the significant progress of multislice spiral chest X-ray technology, more and more acquisition channels are available, and one exposure can 
complete $4,8,16,32$, and 64 . For data acquisition at multiple levels, the utilization of X-rays has increased by the same multiple of the same acquisition level. At the same time, because the scan time is very short, when the pitch is determined to be 3.0 , the radiation dose can be significantly reduced [5]. In general, multislice spiral chest X-ray has significantly improved image quality and scan speed and reduced radiation dose compared with conventional chest $\mathrm{X}$-ray, and its clinical applicability has become wider and wider.

From the results of this study, it is known that poor alveolar inflation and bronchial hyperinflation at all levels are important manifestations of neonatal respiratory distress syndrome, due to the presence of alveolar adhesive atelectasis, which interweaves in the excessively expanded inflatable alveolar ducts and capillary bronchi. Under the influence of reticular shadows, there is an obvious contrast between bronchial levels of normal inflation and atrophic alveoli. It is easy to find inflatable bronchial signs in the middle and late stages of respiratory distress syndrome, and the transparency of the lung field will be significantly reduced after the advanced stage. It has a variety of manifestations and is often called white lung [6-10]. Therefore, it can also be known that, in the clinical diagnosis of neonatal respiratory distress syndrome, the fine granular shadows detected by chest X-ray, reduced lung field transparency, and inflatable bronchial signs can be regarded as reliable signs [9].

\section{Conclusion}

In summary, the early use of NHFV can effectively improve the arterial blood gas analysis of children with RDS and reduce tracheal intubation, operation time, and length of hospital stay. The use of low-dose spiral chest X-ray to diagnose neonatal respiratory distress syndrome is feasible. By reducing the tube current and tube voltage and increasing the pitch, the radiation dose can be reduced while ensuring image quality. By combining clinical manifestations, neonatal breathing can basically achieve the accurate diagnosis of distress syndrome.

\section{Data Availability}

No data were used to support this study.

\section{Conflicts of Interest}

The authors declare that they have no conflicts of interest.

\section{Acknowledgments}

This work was supported by the Key Project of Natural Science of Colleges and Universities in Anhui Province (no. KJ2019A0378).

\section{References}

[1] J. Y. Qiao, Y. Z. Li, H. Y. Wang, and S. D. Zhang, "[As meta analysis of the efficacy of high-frequency oscillatory ventilation versus conventional mechanical ventilation for treating pediatric acute respiratory distress syndrome]," Zhongguo Dang Dai Er Ke Za Zhi, vol. 19, no. 4, pp. 430-435, 2017.

[2] S. Wu, M. Wang, and Y. Zou, "Research on internet information mining based on agent algorithm," Future Generation Computer Systems, vol. 86, pp. 598-602, 2018.

[3] J. Ng and N. D. Ferguson, "High-frequency oscillatory ventilation," Current Opinion in Critical Care, vol. 23, no. 2, pp. 175-179, 2017.

[4] X. W. Zhu, L. P. Shi, L. Liu, and R. Ramanathan, "Non-invasive high-frequency oscillatory ventilation versus nasal continuous positive airway pressure in preterm infants with respiratory distress syndrome: study protocol for a multicenter prospective randomized controlled trial," Trials, vol. 19, no. 1, p. 319, 2018.

[5] J. Marti, P. Hall, P. Hamilton et al., "One-year resource utilisation, costs and quality of life in patients with acute respiratory distress syndrome (ards): secondary analysis of a randomised controlled trial," Journal of Intensive Care, vol. 4, no. 1, p. 56, 2016.

[6] Y.-L. Yang, B.-Q. Wu, J.-Z. Su, L. Yang, and L. Liu, "Clinical efficacy of nasal high-frequency ventilation in treatment of neonatal respiratory distress syndrome: a meta analysis," Zhongguo dang dai er ke za zhi = Chinese journal of contemporary pediatrics, vol. 20, no. 11, pp. 897-903, 2018.

[7] S. A. Ozdemir, E. A. Ozer, O. Ilhan, and S. Sutcuoglu, "Impact of targeted-volume ventilation on pulmonary dynamics in preterm infants with respiratory distress syndrome," Pediatric Pulmonology, vol. 52, no. 2, pp. 213-216, 2016.

[8] S. Wu, "A traffic motion object extraction algorithm," International Journal of Bifurcation and Chaos, vol. 25, no. 14, Article ID 1540039, 2015.

[9] M. Chang, H. Y. Lu, H. Xiang, and H. P. Lan, "[clinical effects of different ways of mechanical ventilation combined with pulmonary surfactant in treatment of acute lung injury/acute respiratory distress syndrome in neonates: a comparative analysis]," Zhonggo Dang Dai Er Ke Za Zhi, vol. 18, no. 11, p. 1069, 2016.

[10] N. Chowdhury, B. L. Giles, and S. D. Dell, "Full-term neonatal respiratory distress and chronic lung disease," Pediatric Annals, vol. 48, no. 4, pp. e175-e181, 2019. 